\title{
Schedulability Analysis of Conditional Parallel Task Graphs in Multicore Systems
}

\author{
Alessandra Melani, Marko Bertogna, Vincenzo Bonifaci, \\ Alberto Marchetti-Spaccamela, and Giorgio Buttazzo
}

\begin{abstract}
Several task models have been introduced in the literature to describe the intrinsic parallelism of real-time activities, including fork/join, synchronous parallel, DAG-based, etc. Although schedulability tests and resource augmentation bounds have been derived for these task models in the context of multicore systems, they are still too pessimistic to describe the execution flow of parallel tasks characterized by multiple (and nested) conditional statements, where it is hard to decide which execution path to select for modeling the worst-case scenario. To overcome this problem, this paper proposes a task model that integrates control flow information by considering conditional parallel tasks (cp-tasks) represented by DAGs with both precedence and conditional edges. For this task model, a set of meaningful parameters are identified and computed by efficient algorithms and a response-time analysis is presented for different scheduling policies. Experimental results are finally reported to evaluate the efficiency of the proposed schedulability tests and their performance with respect to classic tests based on both conditional and non-conditional existing approaches.
\end{abstract}

Index Terms—Parallel scheduling, DAG Tasks, Response-Time Analysis, Multiprocessor Systems, Real-Time Systems.

\section{INTRODUCTION}

$\mathrm{T}$ He availability of multi-/many-core platforms in the embedded market [1], [2], [3], caused an increasing interest for applications with both high-performance and real-time requirements. Following the same trend, several classic schedulability results have been extended to comply with such new platforms, providing new models and tests to guarantee the timing requirements of parallel task systems. Examples of the task models proposed to capture the parallel structure of an application include the fork/join model [21], the synchronous parallel task model [30], and the DAG-based task model [11]. Each of these models divides a task into smaller computational units, called subtasks, which can run simultaneously on different cores.

As noted by Fonseca et al. [20], the problem introduced by conditional statements is particularly significant in the schedulability analysis of parallel tasks running on a multicore system, although explicitly modeling branching structures has been proven useful also for single-core systems to tighten the response time of the tasks [5], [8], [17]. The problem is exacerbated by the presence of multiple conditional statements characterized by branches with very different length. For example, different image processing, object detection/tracking and feature extraction algorithms are characterized by conditional branches with variable sizes. As an example, hierarchical clustering [22] and cascade classifiers [34] are techniques where clusters of pixels are conditionally split/merged depending on image

- A. Melani and G. Buttazzo are with the TeCIP Institute, Scuola Superiore Sant'Anna, Pisa, Italy. E-mail: \{alessandra.melani, g.buttazzo\}@sssup.it.

- M. Bertogna is with the University of Modena and Reggio-Emilia, Modena, Italy. E-mail: marko.bertogna@unimore.it.

- V. Bonifaci is with Istituto di Analisi dei Sistemi ed Informatica, CNR Rome, Italy. E-mail: vincenzo.bonifaci@iasi.cnr.it.

- A. Marchetti-Spaccamela is with Sapienza University of Rome, Rome, Italy.E-mail:alberto@dis.uniroma1.it. features. An efficient implementation of such techniques on a parallel architecture would need to conditionally fork a variable number of parallel sub-tasks depending on runtime information. As a result, the response-time of such a task, as well as its interference on the other tasks, may vary significantly from instance to instance. Therefore, identifying the worst-case scenario that affects system schedulability the most is a challenging issue.

An example of parallel task $T_{0}$ specified according to the OpenMP standard is illustrated in Figure 1. The task has a conditional statement at the beginning of its execution. Depending on the conditional clause, the task can take the upper branch, creating a sequential sub-task $\tau_{1}$ of 10 time units, or the lower branch, forking three sub-tasks $\tau_{2}, \tau_{3}, \tau_{4}$ of 6 time units each. Note that the branch leading to the worst-case response-time depends on the number of cores and the interference from other tasks. For example, with three or more cores and no interfering tasks, the largest response-time is given by the upper branch, for every workconserving scheduler ${ }^{1}$ (i.e., 10 time units instead of 6). With fewer cores, the largest response-time is given by the lower branch (i.e., 12 time units with two cores, and 18 time units with one core). If interfering tasks are present, the situation is even more challenging, because the conclusions derived above may be reversed! For example, adding a sequential task of 6 time-units, the worst-case response-time with three cores is given by the lower branch (12 units instead of 10).

Similarly, it is not easy to predict which branch imposes a larger interference on the other tasks: depending on the characteristics of the other tasks, a higher interference may be produced by a set of parallel sub-tasks or by a longer sequential sub-task. Since applications typically consists of several nested conditional statements, the problem of

1. A scheduler is work-conserving if it never idles a core whenever there is pending workload to execute. 

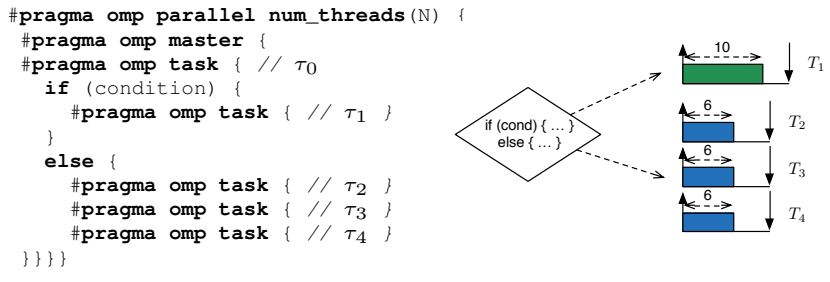

Figure 1: A parallel program with conditional execution.

mapping parallel applications to a task model that does not explicitly consider conditional statements is very difficult to solve.

\subsection{Contributions and paper organization}

This paper extends the parallel DAG model by integrating conditional constructs to provide a tighter analysis to parallel task systems. In the proposed conditional parallel task (cptask) model, each task is represented by a DAG containing both parallel and conditional nodes. To capture the structure of parallel applications, a formal definition of cp-task is provided by specifying the possible connections between the various (conditional and non-conditional) sections of the graph. For the cp-task model, efficient ways to compute an upper-bound on the response-time of each cp-task are derived using different global scheduling algorithms. The effectiveness of the proposed schedulability analysis is assessed by extensive experiments. The paper also shows that the proposed response-time analysis can be efficiently applied to non-conditional task models (such as the DAG task model [11]). For these latter systems, experimental results show that a significantly higher number of schedulable tasksets is detected at a considerably smaller time complexity, with respect to existing approaches.

The remainder of this paper is organized as follows. Section 2 reviews the related work. Sections 3 introduces the proposed task model and the notation used throughout the paper. Section 4 characterizes the critical interference among tasks, while in Section 5 we present our response-time analysis and describe how to compute certain task parameters of interest. Section 6 presents a realistic case study, and then reports our experimental results. The conclusions are drawn in Section 7.

\section{Related Work}

Several parallel task models have been proposed in the realtime literature, although most of them are limited to nonconditional execution modes. One of the first proposals, the fork-join task model, was introduced by Lakshmanan, Kato and Rajkumar [7], [21]. In this model, a task is represented as an alternating sequence of sequential and parallel segments, and every parallel segment has the same degree of parallelism (which is constrained to be less than or equal to the number of available processors). A natural extension of this approach is the synchronous parallel model [6], [18], [26], [27], [30], allowing consecutive parallel segments and an arbitrary degree of parallelism of every segment. Synchronization is still enforced at the boundary of each segment, in the sense that a sub-task in the new segment may start only after all the sub-tasks in the previous segment have completed. A more flexible model of concurrency is the $D A G$ model, where a task is represented by a directed acyclic graph (DAG) in which nodes are sequential subtasks and arcs represent precedence constraints between sub-tasks [9], [11], [15], [23], [24], [25], [29], [31].

The first attempts at enriching a parallel task model with control-flow information were proposed in the context of uniprocessor systems to provide a more accurate characterization of the worst-case behavior of a task [5], [8], [17]. In a multicore setting, Fonseca et al. [20] proposed the multi-DAG model, which represents a parallel task as a collection of DAGs, each representing a different execution flow. The authors proposed a method to combine such flows into a single synchronous parallel task that preserves the execution requirements and the precedence constraints of all the execution flows that can possibly occur at runtime, thus reducing the schedulability problem to a simpler problem for synchronous parallel tasks. A disadvantage of this approach is that it is not scalable with respect to the number of sub-tasks, since the number of different flows through a DAG can be exponential in the number of nodes. Moreover, it adds pessimism in the task transformation process and requires server-based synchronization mechanisms that may be difficult to implement.

The accounting of control flows is common in the field of static program analysis. When detailed control flow information is available, accurate approaches exist that explicitly detect and discard infeasible execution paths, based on the boolean properties tested inside the conditionals. However, infeasible path detection is a difficult problem that in general requires high-complexity machinery, such as the solution of (NP-hard) Satisfiability Modulo Theory instances [14]. Our work, instead, focuses on reasonably fast (at the very least, pseudopolynomial-time) algorithms for schedulability analysis of conditional parallel tasks.

The same conditional parallel task model proposed in this paper was considered by Baruah et al. [10]. However, the authors focused on global Earliest Deadline First (G-EDF) scheduling, proposing an efficient algorithm that transforms any conditional DAG task into a non-conditional DAG task that is "equivalent", in the sense that it preserves the quantities used by the existing tests for DAG tasks without conditional statements [11], [15]. An important difference with our approach is that our analysis method can be applied to any work-conserving scheduler.

Most recently, Baruah [10] extended the federated scheduling paradigm to systems of conditional parallel tasks.

\section{System Model and Definitions}

This paper considers a set $\mathcal{T}=\left\{\tau_{1}, \ldots, \tau_{n}\right\}$ of $n$ sporadic conditional parallel tasks (cp-tasks) that execute upon a platform consisting of $m$ identical processors. Each cp-task $\tau_{k}$ releases a potentially infinite sequence of jobs. Each job of $\tau_{k}$ is separated from the next by at least $T_{k}$ time-units and has a constrained relative deadline $D_{k} \leq T_{k}\left(D_{k}, T_{k} \in \mathbb{N}\right)$. Moreover, each cp-task $\tau_{k}$ is represented as a directed acyclic graph $G_{k}=\left(V_{k}, E_{k}\right)$, where $V_{k}=\left\{v_{k, 1}, \ldots, v_{k, n_{k}}\right\}$ is a 
set of nodes (or vertices) and $E_{k} \subseteq V_{k} \times V_{k}$ is a set of directed arcs (or edges), as shown in Figure 2. Each node $v_{k, j} \in V_{k}$ represents a sequential chunk of execution (or "sub-task") and is characterized by a worst-case execution time $C_{k, j} \in \mathbb{N}$. Preemption/migration overheads and crosscore interference (caused by contention for shared hardware components, such as shared caches, memory buses, etc.) are considered to be negligible. In addition, we assume that $\mathrm{cp}$ tasks do not engage in any synchronization. Arcs represent dependencies between sub-tasks, that is, if $\left(v_{k, 1}, v_{k, 2}\right) \in E_{k}$, then $v_{k, 1}$ must complete before $v_{k, 2}$ can start executing. A node with no incoming arcs is referred to as a source, while a node with no outgoing arcs is referred to as a sink. Without loss of generality, each cp-task is assumed to have exactly one source $v_{k}^{\text {source }}$ and one sink node $v_{k}^{\text {sink }}$. If this is not the case, a dummy source/sink node with zero WCET can be added to the DAG, with arcs to/from all the source/sink nodes. The subscript $k$ in the parameters associated to the task $\tau_{k}$ is omitted whenever the reference to the task is clear in the discussion.

In the cp-task model, nodes can be of two types: a) regular nodes, represented as rectangles, allow all successor nodes to be executed concurrently; b) conditional nodes, coming in pairs and denoted by diamonds and circles, represent the beginning and the end of a conditional construct, respectively, and require the execution of exactly one node among the successors of the start node. The structure of allowed cp-task graphs is formalized in the following recursive definition.

Definition 3.1. A cp-task graph $G$ with source $v^{\prime}$ and sink $v^{\prime \prime}$ is either:

1) (Base case) A (regular) node $v$, with $v=v^{\prime}=v^{\prime \prime}$;

2) (Concurrent composition) A single-source, singlesink DAG obtained from node-disjoint cp-task graphs $G_{1}, \ldots, G_{q}$ (with $v^{\prime}$ the source of $G_{1}, v^{\prime \prime}$ the sink of $G_{q}, q \geq 1$ ) by adding one or more arcs from every sink $v_{i}^{\prime \prime}(i=1, \ldots, q-1)$ to a source $v_{j}^{\prime}$ with $j>i$;

3) (Conditional composition) A single-source, singlesink DAG obtained from node-disjoint cp-task graphs $G_{1}, \ldots, G_{q}$ and from two (conditional) nodes $v^{\prime}, v^{\prime \prime}$ by adding an arc from $v^{\prime}$ to each source $v_{i}^{\prime}$ and from each sink $v_{i}^{\prime \prime}$ to $v^{\prime \prime}(i=1, \ldots, q)$; in this case, $\left(v^{\prime}, v^{\prime \prime}\right)$ is called a conditional pair and each $G_{i}$ a conditional branch.

Example. Figure 2 illustrates a sample cp-task consisting of nine sub-tasks (nodes) $V=\left\{v_{1}, \ldots, v_{9}\right\}$ and twelve precedence constraints (arcs). The number inside each node represents its WCET. Two of the nodes, $v_{2}$ and $v_{6}$, form a conditional pair, meaning that only one sub-task between $v_{3}$ and $v_{4}$ will be executed (but never both), depending on a conditional clause. To see why the graph in Figure 2 satisfies the definition of a cp-task graph, one can reason as follows. The two regular nodes $v_{3}$ and $v_{4}$ fit the base case of the definition; each of them forms a cp-task graph. We conditionally compose them with the conditional pair $\left(v_{2}, v_{6}\right)$ to form a cp-task graph $H$ on nodes $v_{2}, v_{3}, v_{4}, v_{6}$ (with source $v_{2}$ and sink $\left.v_{6}\right)$. Each other regular node $\left(v_{1}\right.$, $\left.v_{5}, v_{7}, v_{8}, v_{9}\right)$ is also a cp-task graph; call $G_{i}$ the graph corresponding to $v_{i}$ for $i \in\{1,5,7,8,9\}$. Finally, we apply concurrent composition to the sequence $G_{1}, G_{5}, H, G_{7}, G_{8}$,

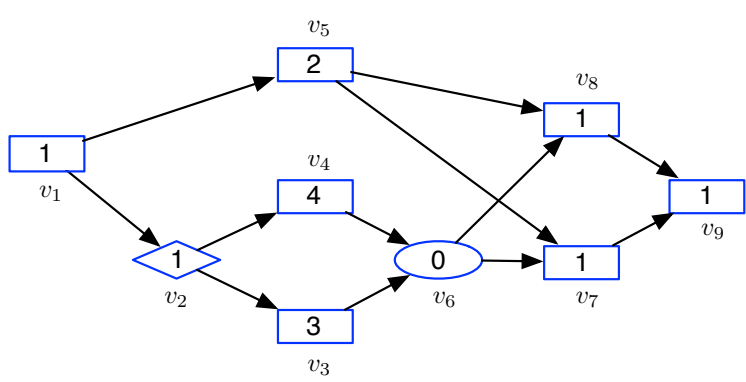

Figure 2: A sample cp-task. Each vertex is labeled with the WCET of the corresponding sub-task.

$G_{9}$ to obtain the cp-task graph in Figure 2. In particular, arcs representing precedence constraints are added from $G_{1}$ to $G_{5}$ and $H$, from $G_{5}$ and $H$ to $G_{7}$ and $G_{8}$, and from $G_{7}$ and $G_{8}$ to $G_{9}$.

We also define a chain or path of a cp-task $\tau_{k}$ as a sequence of nodes $\lambda=\left(v_{k, a}, \ldots, v_{k, b}\right)$ such that $\left(v_{k, j}, v_{k, j+1}\right) \in E_{k}, \forall j \in[a, b)$. The length of a chain of $\tau_{k}$, denoted by $\operatorname{len}(\lambda)$, is the sum of the WCETs of all its nodes, that is, $\sum_{j=a}^{b} C_{k, j}$. A longest path of a cp-task is any source-sink path of the task that achieves the longest length.

Definition 3.2. The length of a cp-task $\tau_{k}$, denoted by $L_{k}$, is the length of any longest path of $\tau_{k}$.

Note that $L_{k}$ also represents the minimum worst-case execution time of cp-task $\tau_{k}$, that is, the time required to execute it when the number of processing units is sufficiently large (potentially infinite) to allow the task to always execute with maximum parallelism. A necessary condition for the feasibility of a cp-task $\tau_{k}$ is thus $L_{k} \leq D_{k}$.

In the absence of conditional branches, the classical sporadic DAG task model defines the volume of the task as the worst-case execution time needed to complete it on a dedicated single-core platform [11], [15], [23], [31]. This quantity can be computed as the sum of the WCETs of all the sub-tasks, that is $\sum_{v_{k, j} \in V_{k}} C_{k, j}$. In the presence of conditional branches, assuming that all sub-tasks are always executed is overly pessimistic. Hence, the concept of volume of a cp-task is generalized by introducing the notion of worst-case workload. Section 5.3 explains in detail how the worst-case workload of a task can be computed efficiently.

Definition 3.3. The worst-case workload $W_{k}$ of a cp-task $\tau_{k}$ is the maximum time needed to execute an instance of $\tau_{k}$ on a dedicated single-core platform, where the maximum is taken among all possible choices of conditional branches.

The utilization $U_{k}$ of a cp-task $\tau_{k}$ is the ratio between its worst-case workload and its period, that is, $U_{k}=W_{k} / T_{k}$. For the task-set $\mathcal{T}$, its total utilization is defined as $U_{\mathcal{T}}=$ $\sum_{i=1}^{n} U_{i}$. A simple necessary condition for feasibility is $U_{\mathcal{T}} \leq m$.

In the example of Figure 2, the length (longest path) is $L=8$, and is given by the chain $\left(v_{1}, v_{2}, v_{4}, v_{6}, v_{7}, v_{9}\right)$. Its volume is 14 units, while its worst-case workload must take into account that either $v_{3}$ or $v_{4}$ are executed at every task instance. Since $v_{4}$ corresponds to the branch with the largest workload, $W=11$. 
Table 1: Notation

\begin{tabular}{c|l||c|l}
\hline \hline $\mathcal{T}$ & set of cp-tasks & $n$ & number of tasks in $\mathcal{T}$ \\
\hline$\tau_{k}$ & $k$-th task of $\mathcal{T}$ & $D_{k}$ & relative deadline of $\tau_{k}$ \\
\hline$T_{k}$ & period of $\tau_{k}$ & $G_{k}$ & DAG associated to $\tau_{k}$ \\
\hline$V_{k}$ & node set of $G_{k}$ & $E_{k}$ & arc set of $G_{k}$ \\
\hline$v_{k, j}$ & $j$ th sub-task of $\tau_{k}$ & $C_{k, j}$ & WCET of $v_{k, j}$ \\
\hline$L_{k}$ & $\begin{array}{l}\text { length of } \tau_{k} \text { 's longest } \\
\text { chain }\end{array}$ & $W_{k}$ & $\begin{array}{l}\text { worst-case workload of } \\
\tau_{k}\end{array}$ \\
\hline$\lambda_{k}^{*}$ & critical chain of $G_{k}$ & $U_{k}$ & utilization of $\tau_{k}$ \\
\hline
\end{tabular}

The notation used throughout the paper is summarized in Table 1.

\section{Critical interference OF CP-TASKS}

This section presents a schedulability analysis for cp-tasks globally scheduled by any work-conserving scheduler. The analysis is based on the notion of interference. In the existing literature for globally scheduled sequential task systems, the interference on a task $\tau_{k}$ is defined as the sum of all intervals in which $\tau_{k}$ is ready, but cannot execute because all cores are busy executing other tasks. We modify this definition to adapt it to the parallel nature of cp-tasks, by introducing the concept of critical interference [18], [26].

Fix a set of cp-tasks $\mathcal{T}$ and a work-conserving scheduler. The first useful notion is that of a critical chain of a task.

Definition 4.1. The critical chain $\lambda_{k}^{*}$ of a cp-task $\tau_{k}$ is the chain of nodes of $\tau_{k}$ that leads to its worst-case response-time $R_{k}$ (in case of ties, fix one such chain arbitrarily).

The critical chain of $\mathrm{cp}$-task $\tau_{k}$ is in principle determined by taking the sink vertex $v_{k}^{\text {sink }}$ of the worst-case instance of $\tau_{k}$ (i.e., the job of $\tau_{k}$ that has largest response-time in the worst-case scenario), and recursively pre-pending the last to complete among the predecessor nodes (whether conditional or not), until the source vertex $v_{k, 1}$ has been included in the chain. A critical node of task $\tau_{k}$ is a node that belongs to $\tau_{k}$ 's critical chain. Since the response-time of a cptask is given by the response-time of the sink vertex of the task, the sink node is always a critical node. For deriving the worst-case response-time of a task, it is then sufficient to characterize the maximum interference suffered by its critical chain.

Definition 4.2. The critical interference $I_{k}$ on task $\tau_{k}$ is defined as the cumulative time during which some critical nodes of the worst-case instance of $\tau_{k}$ are ready, but do not execute because all cores are busy.

Lemma 4.1. Given a set of cp-tasks $\mathcal{T}$ scheduled by any workconserving algorithm on $m$ identical processors, the worst-case response-time $R_{k}$ of each task $\tau_{k}$ satisfies

$$
R_{k} \leq \operatorname{len}\left(\lambda_{k}^{*}\right)+I_{k} .
$$

Proof. Let $r_{k}$ be the release time of the worst-case instance of $\tau_{k}$. In the scheduling window $\left[r_{k}, r_{k}+R_{k}\right]$, the critical chain requires at most $\operatorname{len}\left(\lambda_{k}^{*}\right)$ time-units to complete. By Definition 4.2 , at any time in this window in which $\tau_{k}$ does not suffer critical interference, some node of the critical chain is executing. Therefore $R_{k}-I_{k} \leq \operatorname{len}\left(\lambda_{k}^{*}\right)$.
The difficulty in using Equation (1) for schedulability analysis is that the term $I_{k}$ may not be easy to compute. An established solution is to express the total interfering workload as a function of individual contributions of the interfering tasks, and then upper-bound such contributions with the worst-case workload of each interfering task $\tau_{i}$. In the following, we explain how such interfering contributions can be computed, and how they relate to each other to determine the total interfering workload.

Definition 4.3. The critical interference $I_{i, k}$ imposed by task $\tau_{i}$ on task $\tau_{k}$ is defined as the cumulative workload executed by sub-tasks of $\tau_{i}$ while a critical node of the worst-case instance of $\tau_{k}$ is ready to execute but is not executing.

Lemma 4.2. For any work-conserving algorithm, the following relation holds:

$$
I_{k}=\frac{1}{m} \sum_{\tau_{i} \in \mathcal{T}} I_{i, k} .
$$

Proof. By the work-conserving property of the scheduling algorithm, whenever a critical node of $\tau_{k}$ is interfered, all $m$ cores are busy executing other sub-tasks. The total amount of workload executed by sub-tasks interfering with the critical chain of $\tau_{k}$ is then $m I_{k}$. Hence, $\sum_{\tau_{i} \in \mathcal{T}} I_{i, k}=m I_{k}$, and by reordering the terms, the lemma follows.

Note that when $i=k$, the critical interference $I_{k, k}$ may include the interfering contributions of non-critical subtasks of $\tau_{k}$ on itself, that is, the self-interference of $\tau_{k}$.

By combining Equations (1) and (2), the response-time of a task $\tau_{k}$ can be bounded as

$$
R_{k} \leq \operatorname{len}\left(\lambda_{k}^{*}\right)+\frac{1}{m} I_{k, k}+\frac{1}{m} \sum_{\tau_{i} \in \mathcal{T}, i \neq k} I_{i, k} .
$$

\section{Response-time analysis}

In this section we derive an upper-bound on the worst-case response-time of each cp-task using Equation (3). To this aim we need to bound the interfering contributions $I_{i, k}$. In the sequel, we first consider the inter-task interference $(i \neq k)$ and then the intra-task interference $(i=k)$.

\subsection{Inter-task interference}

We follow the approach adopted in [12], [26], that divides the contribution to the workload of an interfering task $\tau_{i}$ in a window of interest between carry-in, body, and carry-out jobs. The carry-in job is the first instance of $\tau_{i}$ that is part of the window of interest and has release time before and deadline within the window of interest. The carry-out job is the last instance of $\tau_{i}$ executing in the window of interest, having a deadline after the window of interest. All other instances of $\tau_{i}$ are named body jobs.

For sequential task-sets, an upper-bound on the workload of an interfering task $\tau_{i}$ within a window of length $L$ occurs when the first job of $\tau_{i}$ starts executing as late as possible (with a starting time aligned with the beginning of the window of interest) and later jobs are executed as soon as possible [12] (see Figure 3).

For cp-task systems, it is more difficult to determine a configuration that maximizes the carry-in and carry-out contributions. In fact: 


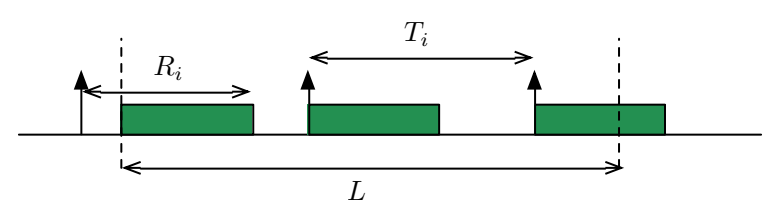

Figure 3: Worst-case scenario to maximize the workload of an interfering task $\tau_{i}$ in the sequential case.

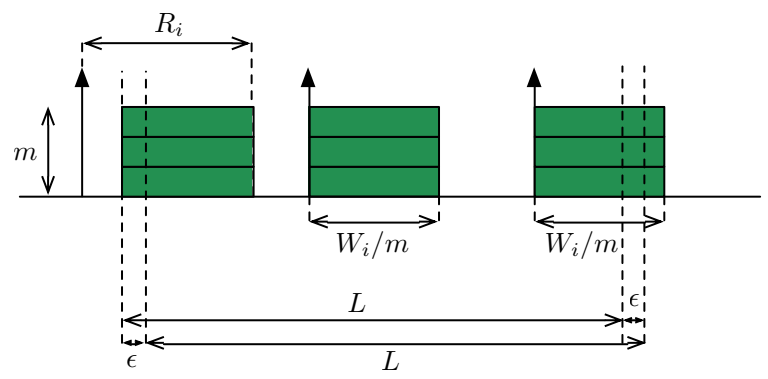

Figure 4: Worst-case scenario to maximize the workload of an interfering cp-task $\tau_{i}$. Shifting the window of interest by $\epsilon$ cannot increase the interfering workload.

1) Due to the precedence constraints and different degree of parallelism of the various execution paths of a cp-task, it may happen that a larger workload is executed within the window if the interfering task is shifted left, i.e., by decreasing the carry-in and increasing the carry-out contributions. This happens for example when the first part of the carry-in job has little parallelism, while the carry-out part at the end of the window contains multiple parallel sub-tasks.

2) A sustainable schedulability analysis [16] must guarantee that all tasks meet their deadlines even when some of them execute less than the worst-case. For example, one of the sub-tasks of an execution path of a cp-task may execute for less than its WCET $C_{i, j}$. This may lead to larger interfering contributions within the window of interest (e.g. a parallel section of a carry-out job is included in the window due to an earlier completion of a preceding sequential section).

3) The carry-in and carry-out contribution of a cp-task may correspond to different conditional paths of the same task, with different levels of parallelism.

To circumvent the above issues, we consider a scenario in which each interfering job of task $\tau_{i}$ executes for its worstcase workload $W_{i}$, i.e., the maximum amount of workload that can be generated by a single instance of a cp-task. We defer the computation of $W_{i}$ to Section 5.3. The next lemma provides a safe upper-bound on the workload of a task $\tau_{i}$ within a window of interest of length $L$.

Lemma 5.1. An upper-bound on the workload of an interfering task $\tau_{i}$ in a window of length $L$ is given by

$$
\mathcal{W}_{i}(L) \stackrel{\text { def }}{=}\left\lceil\left(L+R_{i}-W_{i} / m\right) / T_{i}\right\rceil \cdot W_{i} .
$$

Proof. Consider a situation in which all instances of $\tau_{i}$ execute for their worst-case workload $W_{i}$, evenly distributing the workload among the $m$ cores, as in Figure 4 . The worst-case scenario is obtained when the carry-in job is executed as late as possible, and later jobs execute as soon as they are released, with their minimum inter-arrival time. Consider a problem window of length $L$ aligned with the start of the execution of the carry-in job. Shifting left/right the window by $\epsilon$ cannot possibly increase the contributed workload, nor does it distributing the workload on a lesser number of cores. Consider the enlarged window of length $L+R_{i}-W_{i} / m$ that is aligned with the release of the carryin job: an upper-bound on the number of instances that may execute within such a window is $\left\lceil\left(L+R_{i}-W_{i} / m\right) / T_{i}\right\rceil$, each one contributing for $W_{i}$. This also applies to the (smaller) original window of interest.

When using global EDF, another upper-bound on the interfering contribution of each task is obtained by noting that the deadline of the interfering jobs cannot be later than that of the interfered task.

Lemma 5.2. An upper-bound on the interfering workload of a task $\tau_{i}$ on a task $\tau_{k}$ with global EDF is given by

$$
\mathcal{I}_{i, k} \stackrel{\text { def }}{=}\left\lceil\left(D_{k}-D_{i}+R_{i}\right) / T_{i}\right\rceil \cdot W_{i} .
$$

Proof. Consider a window $\left[r_{k}, r_{k}+D_{k}\right]$ of a task $\tau_{k}$. The interfering contribution of a task $\tau_{i}$ is maximized when the deadline of its carry-out job is aligned with $r_{k}+D_{k}$, and all instances execute as late as possible. Indeed, a later deadline would decrease $\tau_{i}$ 's contribution by a full (carryout) instance, against a potential increase in the carry-in contribution that would not be higher; similarly, an earlier deadline could only decrease the contribution of the previous instances.

Since the response time of $\tau_{i}$ is $R_{i}$, the last instance of $\tau_{i}$ cannot execute between $r_{k}+D_{k}-\left(D_{i}-R_{i}\right)$ and $r_{k}+D_{k}$. We compute the number of jobs that may execute in the window $\left[r_{k}, r_{k}+D_{k}-\left(D_{i}-R_{i}\right)\right]$, which is upper-bounded by $\left\lceil\left(D_{k}-\left(D_{i}-R_{i}\right)\right) / T_{i}\right\rceil$. Since each instance contributes at most $W_{i}$ to the interfering workload, the lemma follows.

\subsection{Intra-task interference}

We now consider the remaining terms of Equation (3), which take into account the contribution of the considered task to its overall response-time, and we compute an upper-bound on $Z_{k} \stackrel{\text { def }}{=} \operatorname{len}\left(\lambda_{k}^{*}\right)+\frac{1}{m} I_{k, k}$.

Lemma 5.3. For a constrained deadline cp-task system scheduled with any work-conserving algorithm, the following relation holds for any task $\tau_{k}$ :

$$
Z_{k}=\operatorname{len}\left(\lambda_{k}^{*}\right)+\frac{1}{m} I_{k, k} \leq L_{k}+\frac{1}{m}\left(W_{k}-L_{k}\right) .
$$

Proof. Since we are in a constrained deadline setting, a job will never be interfered by other jobs of the same task. Recalling that $W_{k}$ is the maximum possible workload produced by a job of cp-task $\tau_{k}$, note that $W_{k} \geq L_{k} \geq \operatorname{len}\left(\lambda_{k}^{*}\right)$. Consider the choice of conditional branches that yields the response time of $\tau_{k}$ and let $W_{k}^{*}$ be the corresponding workload of $\tau_{k}$; then $W_{k}^{*} \leq W_{k}$ by Definition 3.3. Moreover, $I_{k, k}+\operatorname{len}\left(\lambda_{k}^{*}\right) \leq W_{k}^{*}$, since no node in $\lambda_{k}^{*}$ can contribute to $I_{k, k}$ and vice versa. Thus, $I_{k, k} \leq W_{k}-\operatorname{len}\left(\lambda_{k}^{*}\right)$, that is, the portion of $W_{k}$ that may interfere with the critical chain $\lambda_{k}^{*}$ is at most the nonnegative quantity $W_{k}-\operatorname{len}\left(\lambda_{k}^{*}\right)$. Hence,

$$
\operatorname{len}\left(\lambda_{k}^{*}\right)+\frac{1}{m} I_{k, k} \leq \operatorname{len}\left(\lambda_{k}^{*}\right)+\frac{1}{m}\left(W_{k}-\operatorname{len}\left(\lambda_{k}^{*}\right)\right) .
$$


Since len $\left(\lambda_{k}^{*}\right) \leq L_{k}$ and $m \geq 1$, the lemma follows.

Since $Z_{k}$ includes only the contribution of task $\tau_{k}$, one may think that the sum $\left(\operatorname{len}\left(\lambda_{k}^{*}\right)+\frac{1}{m} I_{k, k}\right)$ is equal to the worst-case response-time of $\tau_{k}$ when it is executed in isolation on the multi-core system (i.e., the makespan of $\tau_{k}$ ).

However, this is not true. For example, consider the case of a two-core platform with two cp-tasks $\tau_{k}, \tau_{i}$. Task $\tau_{k}$ has only one if-then-else statement; assume that when the "if" part is executed, the task executes one sub-task of length 10 , otherwise, the task executes two parallel sub-tasks of length 6 each. Thus, the makespan of $\tau_{k}$ is given by the "if" branch, i.e., 10. If $\tau_{i}$ consists of a single sub-task of length 6 , the worst-case response time of $\tau_{k}$ occurs when its "else" branch is executed, yielding a response time of 12 . The share of the response time due to the term $\operatorname{len}\left(\lambda_{k}^{*}\right)+\frac{1}{m} I_{k, k}$ in Equation (3) is $6+(1 / 2) \cdot 6=9$, which is strictly smaller than the makespan. Note that $\operatorname{len}\left(\lambda_{k}^{*}\right)+\frac{1}{m} I_{k, k}$ does not even represent a valid lower bound on the makespan. This can be seen by replacing the "if" branch in the above example with a shorter subtask of length 8 , giving a makespan of 8 . For this reason, one cannot replace the term $\operatorname{len}\left(\lambda_{k}^{*}\right)+\frac{1}{m} I_{k, k}$ in Equation (4) with the makespan of $\tau_{k}$.

The righthand side of Equation (4) has been therefore introduced to upper-bound the term $\operatorname{len}\left(\lambda_{k}^{*}\right)+\frac{1}{m} I_{k, k}$. Interestingly, this quantity does also represent a valid upperbound on the makespan of $\tau_{k}$, i.e., the response time of a cp-task executing in isolation. We omit the proof that is identical to the proofs of the given bounds, considering only the interference due to the task itself.

\subsection{Computation of cp-task parameters}

The upper-bounds on the interference given by Lemmata $5.1,5.2$ and 5.3 require the computation of two characteristic parameters for each cp-task $\tau_{k}$ : the worst-case workload $W_{k}$ and the length of the longest chain $L_{k}$. The longest path of a cp-task can be computed in the same way as for classical DAG task, since any conditional branch defines a set of possible paths in the graph. For this purpose, conditional nodes can be considered as if they were regular nodes. The computation can be implemented in time linear in the size of the DAG by standard techniques (e.g., [19, Section 4.7]).

The computation of the worst-case workload of a cp-task is more involved. We hereafter propose an algorithm to compute $W_{k}$ for each task $\tau_{k}$ in time quadratic in the DAG size; its pseudocode is shown in Algorithm 1. The algorithm first computes a topological order of the $\mathrm{DAG}^{2}$. Then, exploiting the (reverse) topological order, a simple dynamic program can compute for each node the accumulated workload corresponding to the portion of the graph already examined. The algorithm must distinguish the case when the node under analysis is the head of a conditional pair or not. If this is the case, then the maximum accumulated workload among the successors is selected, otherwise the sum of the workload contributions of all successors is computed.

Algorithm 1 takes as input the graph representation of a cp-task $G$ and outputs its worst-case workload $W$. In the

2. A topological order is such that if there is an arc from $u$ to $v$ in the DAG, then $u$ appears before $v$ in the topological order. A topological order can be easily computed in time linear in the size of the DAG (see any basic algorithm textbook, such as [19, Section 3.3.2]).

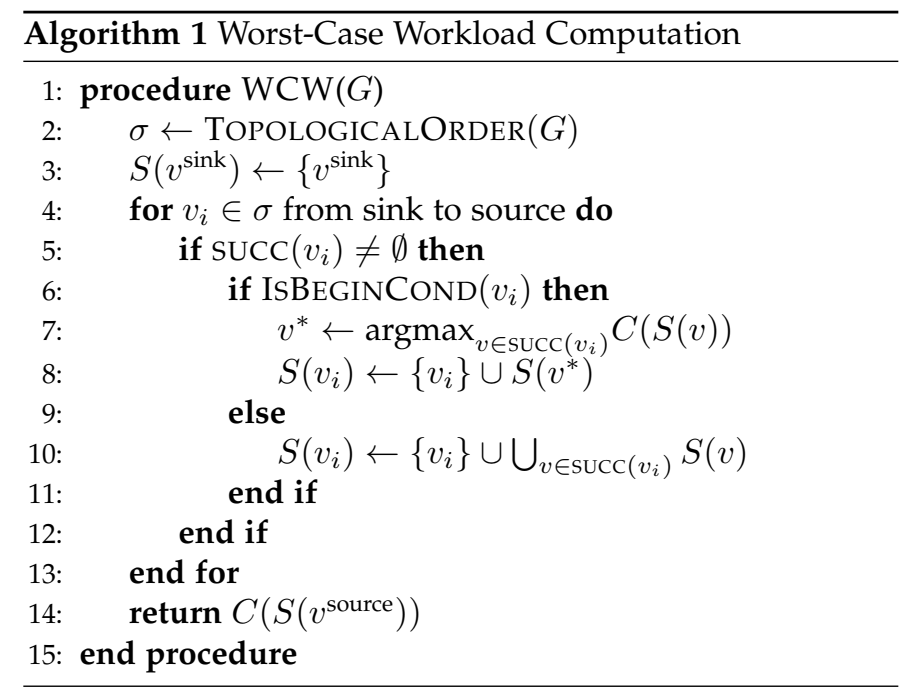

algorithm, for any set of nodes $S$, its total WCET is denoted by $C(S)$. First, at line 2, a topological sorting of the vertices is computed and stored in the permutation $\sigma$. Then, the permutation $\sigma$ is scanned in reverse order, that is, from the (unique) sink to the (unique) source of the DAG. At each iteration of the for loop at line 4 , a node $v_{i}$ is analyzed; a set variable $S\left(v_{i}\right)$ is used to store the set of nodes achieving the worst-case workload of the subgraph including $v_{i}$ and all its descendants in the DAG. Since the sink node has no successors, $S\left(v^{\text {sink }}\right)$ is initialized to $\left\{v^{\text {sink }}\right\}$ at line 3 . Then, the function $\operatorname{SUCC}\left(v_{i}\right)$ computes the set of successors of $v_{i}$. If that set is not empty, function $\operatorname{IsBEGINCOND}\left(v_{i}\right)$ is invoked to determine whether $v_{i}$ is the head node of a conditional pair. If so, the node $v^{*}$ achieving the largest value of $C(S(v))$, among $v \in \operatorname{SUCC}\left(v_{i}\right)$, is computed (line 7). The set $S\left(v^{*}\right)$ therefore achieves the maximum cumulative worstcase workload among the successors of $v_{i}$, and is then used to create $S\left(v_{i}\right)$ together with $v_{i}$. Instead, whenever $v_{i}$ is not the head of a conditional pair, all its successors are executed at runtime. Therefore, the workload contributions of all its successors must be merged into $S\left(v_{i}\right)$ (line 10) together with $v_{i}$. The procedure returns the worst-case workload accumulated by the source vertex, that is $C\left(S\left(v^{\text {source }}\right)\right)$.

The complexity of the algorithm is quadratic in the size of the input DAG. Indeed, there are $O(|E|)$ set operations performed throughout the algorithm, and some operations on a set $S$ (namely, the ones at line 7) also require computing $C(S)$, which has cost $O(|V|)$. So the time complexity is $O(|V||E|)$. To implement the set operations, set membership arrays are sufficient.

One may be tempted to simplify the procedure by avoiding the use of set operations, keeping track only of the cumulative worst-case workload at each node, and allowing a linear complexity in the DAG size. However, such an approach would lead to an overly pessimistic result. Consider a simple graph with a source node forking multiple parallel branches which then converge on a common sink. The cumulative worst-case workload of each parallel path includes the contribution of the sink. If we simply sum such contributions to derive the cumulative worst-case workload of the source, the contribution of the sink would be counted multiple times. Set operations are therefore needed to avoid 
accounting multiple times each node's contribution.

We now present refinements of Algorithm 1 in special sub-cases of interest.

\subsubsection{Non-conditional DAG tasks}

The basic sporadic DAG task model does not explicitly account for conditional branches. Therefore, all vertices of a cp-task contribute to the worst-case workload, which is then equal to the volume of the DAG task: $W_{k}=\sum_{v_{k, j} \in V_{k}} C_{k, j}$. In this particular case, the time complexity to derive the worst-case workload of a task (quadratic in the general case), becomes $O(|V|)$, i.e., linear in the number of vertices.

\subsubsection{Series-parallel conditional DAG tasks}

Some programming languages yield series-parallel cp-tasks, that is, cp-tasks that can be obtained from a single edge by series composition and/or parallel composition. For example, the cp-task in Figure 5 is series-parallel, while the cp-tasks in Figures 2 and 6 are not. Such a structure can be detected in linear time [32]. In series-parallel graphs, for every head $s_{i}$ of a conditional or parallel branch there is a corresponding tail $t_{i}$. For example, in Figure 5, the tail corresponding to parallel branch head $v_{2}$ is $v_{9}$. Algorithm 1 can be specialized to series-parallel graphs. For each vertex $u$, the algorithm will simply keep track of the worst-case workload of the subgraph reachable from $u$, as follows. For each head vertex $s_{i}$ of a parallel branch, the contribution from all successors should be added to $s_{i}$ 's WCET, subtracting however the worst-case workload of the corresponding tail $t_{i}$ a number of times equal to the out-degree of $s_{i}$ minus 1 ; for each head vertex $s_{i}$ of a conditional branch, only the maximum among the successors' worst-case workloads is added to $s_{i}$ 's WCET. Finally, for all non-head vertices add the worst-case workload of their unique successor to their WCET. The complexity of this algorithm reduces then to $O(|E|)$, i.e., it becomes linear in the size of the graph.

\subsection{Schedulability condition}

Lemmata 5.1 and 5.3 and the bounds previously computed allow proving the following theorem [12].

Theorem 5.1. Given a cp-task set globally scheduled on $m$ cores, an upper-bound $R_{k}^{u b}$ on the response-time of a task $\tau_{k}$ can be derived by the fixed-point iteration of the following expression, starting with $R_{k}^{u b}=L_{k}$ :

$$
R_{k}^{u b} \leftarrow L_{k}+\frac{1}{m}\left(W_{k}-L_{k}\right)+\frac{1}{m} \sum_{\forall i \neq k} \mathcal{X}_{i}^{A L G},
$$

where, with global FP:

$$
\mathcal{X}_{i}^{A L G}=\mathcal{X}_{i}^{F P}=\left\{\begin{array}{lr}
\mathcal{W}_{i}\left(R_{k}^{u b}\right), & \forall i<k \\
0, & \text { otherwise }
\end{array} ;\right.
$$

with global EDF:

$$
\mathcal{X}_{i}^{A L G}=\mathcal{X}_{i}^{E D F}=\min \left\{\mathcal{W}_{i}\left(R_{k}^{u b}\right), \mathcal{I}_{i, k}\right\} ;
$$

and $\mathcal{X}_{i}^{A L G}=\mathcal{W}_{i}\left(R_{k}^{u b}\right)$ for any work-conserving scheduler.

The schedulability of a cp-task system can then be simply checked using Theorem 5.1 to compute an upper-bound on the response-time of each task. In the FP case, the bounds

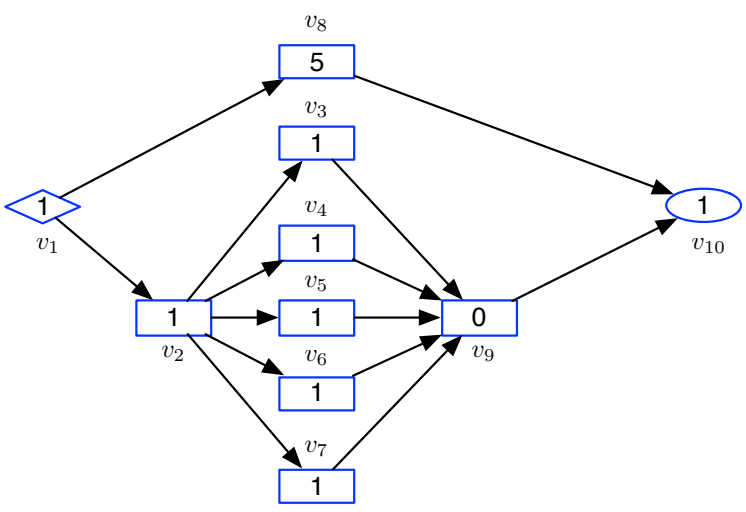

Figure 5: Example of cp-task that shows the pessimism of the upper-bound given in Equation (4).

are updated in decreasing priority order, starting from the highest priority task. In this case, it is sufficient to apply Theorem 5.1 only once for each task. Instead, in the EDF or general work-conserving cases, multiple rounds may be necessary. All bounds are initially set to $R_{k}^{u b}=L_{k}, \forall \tau_{k} \in \mathcal{T}$. Then, Theorem 5.1 is used to compute a response-time bound for each task $\tau_{k}$. The procedure continues until either (i) one of the response-time bounds exceeds the corresponding task deadline (returning a negative schedulability result), or (ii) a fixed-point is reached (returning a schedulable condition).

Since $\mathcal{W}_{i}$ is a step function of $R_{k}^{u b}$, each iteration increases $R_{k}^{u b}$ by at least $1 / m$, but never beyond $D_{k}$. Therefore, the test converges within a pseudopolynomial number of steps.

\subsection{Improved upper-bounds on intra-task interference}

The upper bound given in Lemma 5.3 might be pessimistic. As an example, consider the cp-task $\tau_{k}$ in Figure 5, which executes on a platform composed of $m=2$ processors. This cp-task has a longest path length of 7 time-units (given by the upper branch), and a worst-case workload $W_{k}=8$ timeunits (given by the lower branch). When $m=2$, Equation (4) gives a bound on $Z_{k}$ of 7.5. However, if the upper branch is taken after the completion of $v_{1}$, only the longest path of $\tau_{k}$ would be executed, yielding a value of $Z_{k}=7$ time-units. Instead, if the lower branch is taken, only the corresponding portion of the graph would be executed, with an upperbound of $Z_{k} \leq 4+4 / 2=6$ time-units. Hence, in both cases, the upper-bound computed by (4) would be pessimistic.

This is mainly due to the fact that Equation (4) considers the worst-case situation where, simultaneously, i) the critical path of $G_{k}$ is executed; and ii) the total worst-case workload of $\tau_{k}$ is experienced. However, given the internal structure of the cp-task of Figure 5, this situation can never happen.

The example intuitively suggests that the bound in Equation (4) can be further improved by jointly computing the worst-case workload and the longest chain length for each portion of the cp-task, so that both values refer to the same conditional branch. Specifically, for a given chain $\lambda$ of $\tau_{k}$, let $W_{k}^{\lambda}$ be the maximum workload attainable by those instances of $\tau_{k}$ in which all nodes in $\lambda$ are executed. 
Then, arguing similarly as in Lemma 5.3, we get:

Lemma 5.4.

$$
\begin{aligned}
Z_{k} & \leq \operatorname{len}\left(\lambda_{k}^{*}\right)+\frac{1}{m}\left(W_{k}^{\lambda_{k}^{*}}-\operatorname{len}\left(\lambda_{k}^{*}\right)\right) \\
& \leq \max _{\lambda}\left(\operatorname{len}(\lambda)+\frac{1}{m}\left(W_{k}^{\lambda}-\operatorname{len}(\lambda)\right)\right)
\end{aligned}
$$

where $\lambda$ ranges over all source-sink paths of $\tau_{k}$.

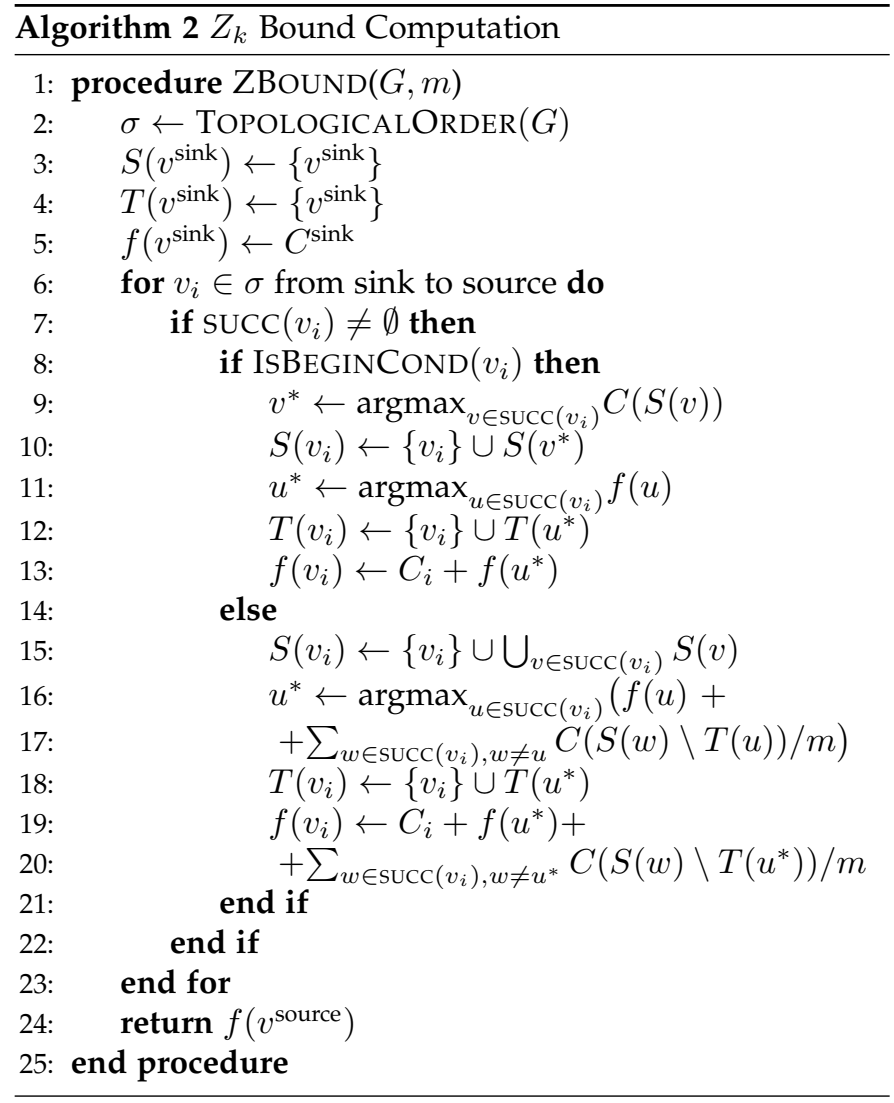

Algorithm 2 takes as input a given task graph $G$ and $m$ and outputs an upper-bound on the task's $Z_{k}$ value by computing jointly the worst-case workload and the contributions of different subgraphs of the task. As for Algorithm 1 , a topological sorting of the nodes is required (line 2). Three variables for each node $v_{i}$ are used by the algorithm to store intermediate results: $S\left(v_{i}\right)$ is a set representing the nodes that determine the largest partial workload from $v_{i}$ till the end of the DAG; $f\left(v_{i}\right)$ stores the bound on the partial $Z_{k}$ value from node $v_{i}$ to the end of the DAG, including the full contribution of nodes belonging to the partial longest chain (stored in set $T\left(v_{i}\right)$ ) and the workload contribution over $m$ cores due to other nodes of the same conditional instance. The computation of the values $S\left(v_{i}\right)$ (lines 3, 10, $15)$ is exactly as in Algorithm 1.

In the sequel, we focus on the computation of $f\left(v_{i}\right)$ and $T\left(v_{i}\right)$. Since the sink node has no successors, we initialize $T\left(v^{\text {sink }}\right)$ to $\left\{v^{\text {sink }}\right\}$ and $f\left(v^{\text {sink }}\right)$ to $C^{\text {sink }}$. The algorithm's main loop iterates over the nodes of $G$ in reverse topological order (line 6). If the node under analysis has some successor, different actions are taken depending on whether $v_{i}$ is the head of a conditional pair or not. In the former case, we compute the successor $u^{*}$ that maximizes the intermediate

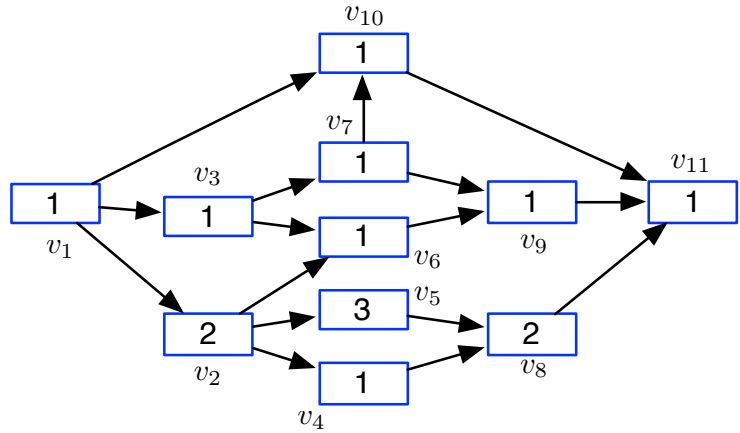

Figure 6: Example of cp-task that shows the two sources of pessimism in the upper-bound of Algorithm 2.

upper-bound on $Z_{k}$, and set $f\left(v_{i}\right)$ and $T\left(v_{i}\right)$ accordingly. If, instead, a parallel branch is departing from the current node $v_{i}$, the workload by all the successors will be transferred to $v_{i}$. The goal is to determine the successor $u$ that yields the largest combined value of its partial $Z_{k}$ bound $(f(u))$ plus the total self-interference from other nodes, which is bounded by

$$
\sum_{w \in \operatorname{Succ}\left(v_{i}\right), w \neq u} C(S(w) \backslash T(u)) / m .
$$

Note that the set $T(u)$ is subtracted from the set to consider for the self-interfering contribution, because such nodes are already fully accounted for in the term $f(u)$.

The complexity of Algorithm 2 is $O(|V||E| \Delta)$, where $\Delta$ denotes the maximum out-degree of a node. In fact, similarly to the analysis of Algorithm 1, the complexity of the algorithm is $O(|V||E|)$, plus the cost of executing the instructions at lines $16-17$. The cost of performing such an instruction once is $O\left(|V| \delta\left(v_{i}\right)^{2}\right)$, where $\delta\left(v_{i}\right)$ is the outdegree of node $v_{i}$; since $\delta\left(v_{i}\right) \leq \Delta$, it follows that the total cost of the instructions at line $16-17$ is

$$
O\left(|V| \sum_{i} \delta\left(v_{i}\right)^{2}\right)=O\left(|V| \Delta \sum_{i} \delta\left(v_{i}\right)\right)=O(|V||E| \Delta) .
$$

This cost dominates, in the worst case, the cost of other operations; hence, the complexity of Algorithm 2 is $O(|V||E| \Delta)$.

\subsubsection{A non-redundant upper-bound}

The previously introduced upper-bound is still not accurate, because it may lead to account multiple times the interfering contribution of some vertices. In Figure 6 we report an example to intuitively show the two sources of pessimism that affect Algorithm 2. In this example, we assume that the cp-task $\tau_{k}$ in Figure 6 executes on $m=2$ processors.

When Algorithm 2 examines vertex $v_{2}$, it designates $v_{5}$ as the vertex $u^{*}$ that maximizes its partial bound on $Z_{k}$ plus the intra-task interference from the other successors. In particular, the instruction at lines 19-20 yields

$$
\begin{aligned}
f\left(v_{2}\right) & =C_{2}+f\left(v_{5}\right)+\frac{1}{m}\left(C\left(S\left(v_{4}\right) \backslash T\left(v_{5}\right)\right)+\right. \\
& \left.+C\left(S\left(v_{6}\right) \backslash T\left(v_{5}\right)\right)\right)=2+6+(1+2) / 2=9.5 .
\end{aligned}
$$


When vertex $v_{1}$ is examined, the algorithm selects $v_{2}$ as the vertex $u^{*}$, and the instruction at lines 19-20 now yields

$$
\begin{aligned}
f\left(v_{1}\right) & =C_{1}+f\left(v_{2}\right)+\frac{1}{m}\left(C \left(S\left(v_{3}\right) \backslash T\left(v_{2}\right)+\right.\right. \\
& +C\left(S\left(v_{10}\right) \backslash T\left(v_{2}\right)\right)=1+9.5+(5+1) / 2=13.5,
\end{aligned}
$$

which also represents the final output of the algorithm. Note, however, that the contribution of vertices $v_{6}$ and $v_{9}$ has been accounted twice: first, as intra-task interference on $v_{2}$ (i.e., inside set $S\left(v_{6}\right) \backslash T\left(v_{5}\right)$ in Equation (6)), and then as intra-task interference on $v_{1}$ (i.e., inside set $S\left(v_{3}\right) \backslash T\left(v_{2}\right)$ ) in Equation (7). Analogously, also the contribution of $v_{10}$ is accounted twice by Algorithm 2: first, among the successors of $v_{7}$, and then, among the successors of $v_{1}$. We identify two sources of pessimism in Algorithm 2.

First source of pessimism. When examining vertex $u$, vertices reachable from successors of $u$ (other than $u^{*}$ ) that have already been considered when examining $u^{*}$ may be counted multiple times (as $v_{6}$ and $v_{9}$ in the example above).

This problem can be overcome by replacing the summation at lines 17 and 20 of Algorithm 2 with the expression

$$
\sum_{w \in \operatorname{Succ}\left(v_{i}\right), w \neq u} C(S(w) \backslash S(u)) / m
$$

The new expression differs from the one given in Algorithm 2 because it subtracts $S(u)$ (instead of $T(u)$ ) from the set to consider for the self-interfering contribution, since such vertices have been already fully accounted for in the term $f(u)$, either as part of the partial longest chain or as intratask interference on $u$. Since the set $S(u)$ contains vertices from both sets, the new formulation allows discarding all vertices that have already been accounted when examining $u^{*}$. Indeed, applying this improvement to the above example, the contribution from vertices in $S\left(v_{3}\right) \backslash S\left(v_{2}\right)$ will be computed (instead of $S\left(v_{3}\right) \backslash T\left(v_{2}\right)$ ) and added to $f\left(v_{1}\right)$; since $S\left(v_{2}\right)$ also contains $v_{6}$ and $v_{9}$, their contribution will not be counted multiple times, leading to a tighter bound on $Z_{k}$ given by

$$
\begin{aligned}
f\left(v_{1}\right) & =C_{1}+f\left(v_{2}\right)+\frac{1}{m}\left(C\left(S\left(v_{3}\right) \backslash S\left(v_{2}\right)\right)+\right. \\
& +C\left(S\left(v_{10}\right) \backslash S\left(v_{2}\right)\right)=1+9.5+(3+1) / 2=12.5
\end{aligned}
$$

Second source of pessimism. When examining vertex $u$, vertices reachable from successors of $u$ (other than $u^{*}$ ) that have been previously examined may be accounted multiple times (as vertex $v_{10}$ in the example above).

This second issue can be prevented by using set operations instead of the sum operand at lines 17 and 20 of Algorithm 2, which leads to the expression

$$
\begin{aligned}
\frac{1}{m} & \cdot C\left(\bigcup_{w \in \operatorname{succ}\left(v_{i}\right), w \neq u} S(w) \backslash S(u)\right)= \\
& =\frac{1}{m} \cdot C\left(S\left(v_{i}\right) \backslash S(u) \backslash\left\{v_{i}\right\}\right) .
\end{aligned}
$$

Applying this refined expression to the example above, the contribution of $v_{10}$ is finally accounted only once. It can be easily verified that the final bound on $Z_{k}$ is now 12 .

The following lemma proves that with the improvements to Algorithm 2 discussed above, the bound on $Z_{k}$

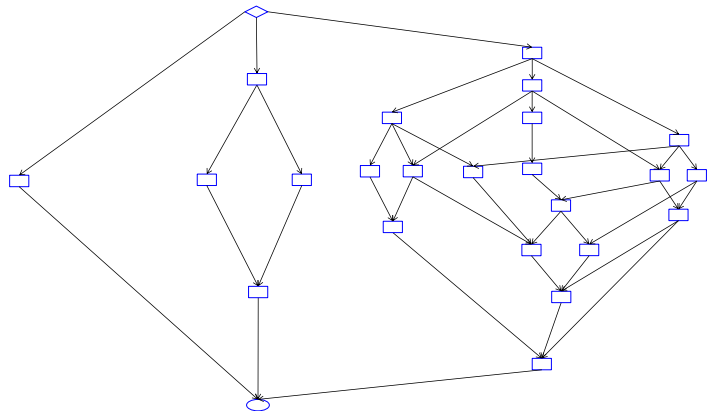

Figure 7: Graph structure of the Cholesky benchmark.

that we obtain is non-redundant, in the sense that it does not account the contribution of any vertex more than once.

Lemma 5.5. Algorithm 2 with the improvements discussed above yields a non-redundant upper-bound on $Z_{k}$.

Proof. The only steps in the updated algorithm where the contribution of a vertex may be added more than once are line 13 and lines 19-20. At line 13, only the contributions of a vertex $v_{i}$ and of some of its descendants (those in $S\left(u^{*}\right) \subset$ $\left.S\left(v_{i}\right) \backslash\left\{v_{i}\right\}\right)$ are being added, but $v_{i} \notin S\left(u^{*}\right)$, so the two sets are disjoint. At lines 19-20, a vertex $v_{i}$, and some of its descendants in $S\left(u^{*}\right)$ (line 19) and in $S\left(v_{i}\right) \backslash S\left(u^{*}\right) \backslash\left\{v_{i}\right\}$ (the updated line 20) are being considered. Again, these sets are disjoint and no double counting can occur.

With a reasoning similar to the one in Section 5.5 one obtains that the complexity of the updated Algorithm 2 is

$$
O\left(|V| \sum_{i} \delta\left(v_{i}\right)|V|\right)=O\left(|V|^{2} \sum_{i} \delta\left(v_{i}\right)\right)=O\left(|V|^{2}|E|\right) .
$$

\section{Experimental Characterization}

In order to evaluate the effectiveness of the proposed approach, we faced the problem of generating a large number of conditional parallel task sets that are representative of realistic workloads. To do that, we (i) selected three real parallel programs with sufficiently different topologies, implemented in OpenMP; (ii) extracted and characterized their cp-DAG structures; and (iii) developed a (non-trivial) graph generation tool that may reproduce the structures of the considered programs. The parallel programs that have been selected are:

- Wavefront, a matrix processing algorithm in which the matrix is decomposed into smaller blocks and traversed through its diagonals. The program takes as input the block size $b s$;

- Cholesky, a factorization algorithm used for efficient numerical solution of systems of linear equations or Monte Carlo simulation techniques. The program takes as input the number of blocks $n b$ per dimension, and performs the factorization on block submatrices;

- $E S A$, an infrared pre-processing application developed by the European Space Agency (ESA) and used by H2RG sensors to measure the red-shift of galaxies. 
To determine the cp-task structures corresponding to the considered OpenMP programs, we adopted a tool, presented in [33], that takes as input an OpenMP program and extracts the corresponding DAG structure. The considered OpenMP benchmarks have been executed on a Intel(R) Core(TM) i7-4600U processor, with 4 cores and 2 hardware threads per core, and a $6 \mathrm{MB}$ L3 cache, and then processed by the tool in [33] to extract the corresponding cp-DAGs. For the first two benchmarks (Wavefront and Cholesky), we consider a restricted input set, namely $b s \in\{1,2,4\}$ and $n b \in\{1,2,4\}$, respectively. Different computation is performed by the two programs depending on the input value, hence their corresponding cp-DAGs have a conditional head node and three branches with significantly unbalanced workload (see Figure 7). Conversely, ESA is a non-conditional (i.e., classic) DAG with a very high level of parallelism and a large degree of connectivity.

Finally, we implemented a cp-task generation tool that could reproduce the different structures of the selected use cases. We refer to [28] as a baseline for our random cp-task generation. In that work, multiple nested levels of conditional branches are recursively generated by expanding nonterminal vertices (called blocks) either to terminal vertices or to conditional subgraphs, until reaching a maximum recursion depth. The derivation rules given in [28] need to be extended by considering that non-terminal vertices can be expanded to either terminal vertices, parallel subgraphs or conditional subgraphs. We specify the probabilities that control such three events as $p_{\text {term }}, p_{\text {par }}$, and $p_{\text {cond }}$, respectively, requiring that $p_{\text {term }}+p_{\text {par }}+p_{\text {cond }}=1$.

Additionally, the maximum number of branches of parallel and conditional subgraphs are indicated as $n_{p a r}$ and $n_{\text {cond }}$, respectively. Hence, whenever a parallel subgraph is generated, the number of its branches is uniformly selected in the interval $\left[2, n_{\text {par }}\right]$. Analogously, whenever a nonterminal vertex is expanded to a conditional subgraph, the number of branches is uniformly selected in $\left[2, n_{\text {cond }}\right]$.

Unfortunately, this methodology only allows generating series-parallel graphs. Therefore, since in this work we deal with a more general class of task graphs (i.e., cp-DAGs that respect the structural restrictions imposed by conditional nodes, as described in Section 3), we place additional edges between pairs of nodes to obtain cp-DAG tasks. In our methodology, an edge is added between two vertices with a certain probability $p_{a d d}$, provided that any of such edges respects the structural restrictions dictated by our cp-task model (see Definition 3.1).

Each cp-task $\tau_{k}$ is generated as follows:

- the WCET $C_{k, j}$ of each vertex $v_{k, j}$ is randomly selected as a positive integer in the interval $[1,100]$;

- then, $L_{k}$ and $W_{k}$ are computed;

- the period $T_{k}$ is uniformly selected as an integer in the interval $\left[L_{k}, W_{k} / \beta\right]$, where $\beta \leq 1$ is a parameter that controls the minimum cp-task utilization. In this way, the utilization of each cp-task is uniformly distributed in the interval $\left[\beta, W_{k} / L_{k}\right]$, where its right endpoint, corresponding to the maximum possible utilization, is given by the average degree of parallelism of the cp-task;
- finally, the relative deadline $D_{k}$ is uniformly selected as an integer in the interval $\left[L_{k}, T_{k}\right]$.

Whenever a specific utilization is targeted, we repeatedly add tasks until the desired cumulative utilization is exceeded. Then, the period of the last task is increased to match the desired total system utilization. In all our experiments, we set the maximum recursion depth to 3 for each cp-task.

The synthetic cp-task generator described above is able to generate workloads that closely match the considered real graph structures. In particular, Figure 8 illustrates a randomly generated graph that resembles the structure of Wavefront and Cholesky (with a conditional head node and three branches with highly unbalanced workload). The graph has been obtained by setting $n_{\text {cond }}=3, n_{\text {par }}=6$, $p_{\text {cond }}=0.4, p_{\text {par }}=0.4, p_{\text {term }}=0.2, p_{\text {add }}=0.1$. Analogously, Figure 9 shows a strongly connected and highly parallel DAG resembling the ESA benchmark, obtained by setting $n_{\text {par }}=10, p_{\text {cond }}=0, p_{\text {par }}=0.8, p_{\text {term }}=0.2$, $p_{\text {add }}=0.1$.

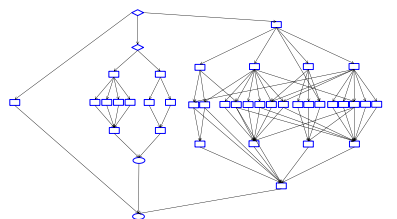

Figure 8: Randomly generated cp-task having three conditional branches with unbalanced workload.

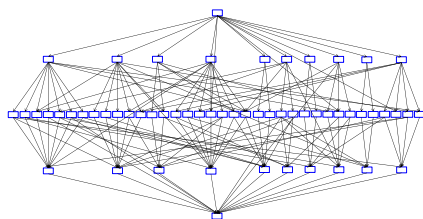

Figure 9: Randomly generated cp-task with a high level of parallelism and a large degree of connectivity.

\subsection{Experimental results for $\mathrm{cp}$-tasks}

We show the experimental comparison of our response-time analysis against the only two works in the literature that target the global scheduling of DAG tasks with conditional branches, i.e., [20] and [10]. The first work proposes a transformation of conditional DAG tasks scheduled with global FP into synchronous parallel tasks. Existing schedulability tests can then be applied on the transformed task-set. For this purpose, we adopted the test for synchronous parallel tasks proposed by Maia et al. [26], which, to the best of our knowledge, outperforms the others. This schedulability test will be referred to as COND-SP. The second work by Baruah et al. [10] proposes a method to transform any conditional DAG task into a non-conditional DAG task on which the existing tests in [15] and [9] can be applied. Since the test in [9] analytically dominates the one in [15], we will only use the former in our comparison. Finally, we will denote as RTA-XXX-a (resp., RTA-XXX-b) our response-time analysis for FP $(X X X=F P)$ or EDF $(X X X=E D F)$ when the intra-task interference is bounded as described in Algorithm 2, with (resp., without) the improvements discussed in Section 5.5.1. All the algorithms compared in our experiments have been implemented as MATLAB $^{\circledR}$ code. Since a considerable effort was required to design a sufficiently general setting for evaluating the performance of graph-based task systems, we created a public repository [4] where our code can be 
Table 2: Case study description

\begin{tabular}{c||c|c|c|c|c} 
Benchmark & $\mathbf{L}_{\mathbf{k}}$ & $\mathbf{W}_{\mathbf{k}}$ & $\mathbf{D}_{\mathbf{k}}$ & $\mathbf{T}_{\mathbf{k}}$ & $\mathbf{p}_{\mathbf{k}}$ \\
\hline \hline Wavefront & 1635 & 3252 & 2000 & 2600 & High \\
\hline ESA & 5784 & 48075 & 17600 & 22000 & Medium \\
\hline Cholesky & 1664 & 3812 & 17000 & 25000 & Low \\
\hline
\end{tabular}

freely downloaded to test the schedulability performance of conditional/parallel task systems.

In the first experiment, we considered an application composed of the three representative programs, setting deadlines and periods to produce a non-trivial scheduling scenario. Table 2 describes for each program its critical path length $L_{k}$, worst-case workload $W_{k}$, relative deadline $D_{k}$, period $T_{k}$ and static priority level $p_{k}$. Time values are given in microseconds.

Our objective is to determine what is the minimum number of processors required to successfully schedule the considered application. Under global FP scheduling, the task-set described above can be successfully scheduled on a platform consisting of $m=6$ processors, being $R_{1}=$ $1904.5 \leq D_{1}, R_{2}=16626 \leq D_{2}$ and $R_{3}=13286 \leq D_{3}$. Conversely, when using COND-SP, $m=11$ processors are required to schedule the given application. Hence, our test is able to almost halve the required computational resources. Also, notice that the task-set in Table 2 would not be deemed schedulable by RTA-FP on $m=6$ processors if a Deadline Monotonic priority ordering was considered ( $m=7$ processors would be necessary in that case). If, instead, global EDF scheduling is assumed, our RTA-EDF approach requires $m=8$ processors to deem the task-set schedulable, which is the same result obtained when using the approach by Baruah.

To give a larger perspective of the relative performance of the considered algorithms, we hereafter show the number of schedulable task-sets detected by each algorithm among the tasks randomly generated with our tool. For each experiment, 1000 task-sets are newly generated for each value on the $x$-axis.

We first consider the FP case, with tasks priorities assigned according to a Deadline Monotonic (DM) ordering, setting $p_{\text {cond }}=0.4, p_{\text {par }}=0.4, p_{\text {term }}=0.2, p_{\text {add }}=0.1$, $n_{\text {cond }}=2, n_{\text {par }}=6, \beta=0.1$.

In the first set of experiments, we varied the total system utilization $U_{\mathcal{T}}$ in the range $[0, m]$. The number of schedulable task-sets obtained when $m=4$ is reported in Figure 10. The trend observed in the figure, which is representative of the general behavior, shows that RTA-FP clearly outperforms COND-SP for any value of $U_{\mathcal{T}}$.

In the second set of experiments, we varied the number of processors. The results for $U_{\mathcal{T}}=2$ are reported in Figure 11. For low values of $m$, RTA-FP significantly outperforms COND-SP, while for a large number of processors both tests are able to successfully schedule nearly all task-sets.

In the third set of experiments, the number of tasks $n$ is varied in the range $[1,20]$. Since $n$ has now a fixed value in each experiment, individual cp-task utilizations have been computed using UUnifast [13]. Figure 12 illustrates the results for $m=4$ and $U_{\mathcal{T}}=2$. While the performance of the two tests is comparable when $n$ is small, RTA-FP exhibits a substantial improvement over COND-SP when $n \geq 4$. Furthermore, RTA-FP achieves full schedulability for large values of $n$. This conforms to the intuition that scheduling a large number of "light" tasks is easier than scheduling fewer "heavy" tasks. Instead, COND-SP achieves its maximum schedulability performance for $n=5$ and then degrades for higher values of $n$. This stems from the pessimism introduced by the transformation technique in [20], which increases when the number of tasks is higher.

Another drawback of COND-SP concerns its complexity, since it requires enumerating all the conditional flows of each cp-task, which are exponentially many in the nesting level of conditional statements. Instead, our approach relies on efficient algorithms that explicitly deal with conditional branches in psuedo-polynomial time. The main consequence is that the running time of COND-SP is often quite prohibitive, while RTA-FP runs very fast (i.e., in the order of milliseconds).

Other experiments have been performed by varying the connectivity degree of the tasks, i.e., by changing the probability $p_{a d d}$, while keeping $U_{\mathcal{T}}, n$ and $m$ constant. However, the results do not show any particular trend, as the schedulability ratio remains almost constant for all possible values of $p_{\text {add }}$, hence the corresponding plots are not reported. In other experiments, we have also varied the composition of the cp-tasks, by acting on $p_{\text {cond }}$ and $p_{\text {par }}$, while keeping their sum constant. Again, no interesting trend has been identified. Such results can be explained considering that the computation of the interference produced by each $\mathrm{cp}$ task highly depends on its worst-case workload, and its computation (see Algorithm 1) is not very much influenced by the degree of parallelism of the cp-task. Furthermore, the notion of worst-case workload represents an effective way of abstracting from the different conditional flows. This explains why the composition of tasks in terms of conditional/parallel branches does not significantly affect the schedulability performance.

We now proceed to the global EDF case. In Figure 13, we report the number of schedulable task-sets with $m=4$ when $U_{\mathcal{T}} \in[0,4]$. At all utilization levels, our approach is able to successfully schedule more task-sets. The same trend can be observed when varying the number of cores. Figure 14 illustrates representative results when $U_{\mathcal{T}}=2$ and $m \in[2,30]$. Our approach performs significantly better for any value of $m$ : in particular, while RTA-EDF is able to schedule nearly all task-sets for a large value of $m$, the approach by Baruah et al. cannot admit a large share of the generated task-sets even when the number of cores significantly increases.

Finally, Figure 15 shows the comparison between the two approaches when varying the number of tasks $(n \in[1,20])$, with $U_{\mathcal{T}}=2$ and $m=8$. While RTA-EDF admits almost all task-sets when $n \geq 7$, the approach by Baruah et al. achieves full schedulability only for a very large value of $n$.

\subsection{Experimental results for classic DAG tasks}

The following experiments restrict our task model to the case where conditional statements are not modeled, i.e., classic DAG tasks are considered. This particular setting allowed us to evaluate the improvement of our response- 


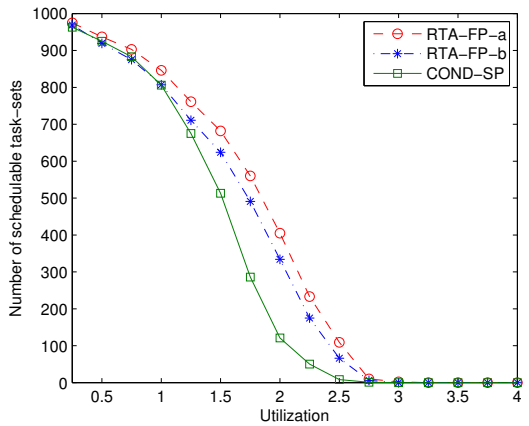

Figure 10: RTA-FP as a function of $U_{\mathcal{T}}$ ( $m=4$, constrained deadlines).

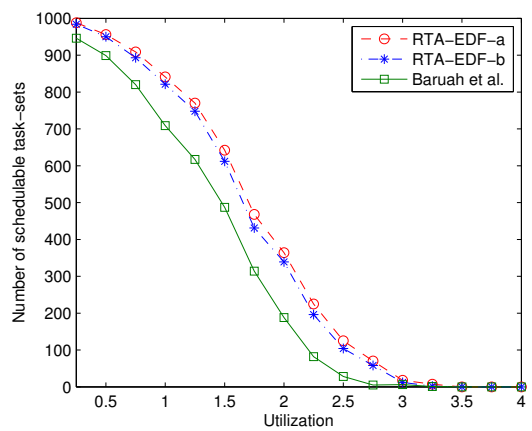

Figure 13: RTA-EDF as a function of $U_{\mathcal{T}}$ ( $m=8$, constrained deadlines).

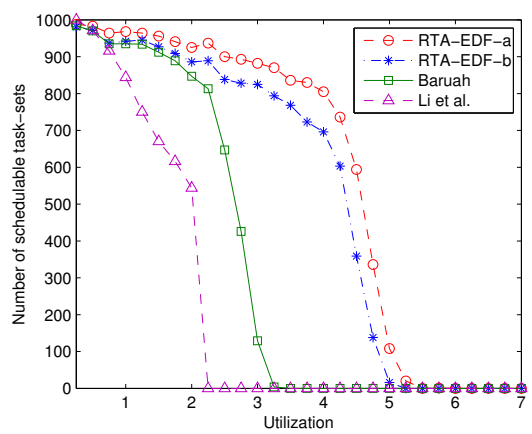

Figure 16: RTA-EDF as a function of $U_{\mathcal{T}}$ ( $m=8$, implicit deadlines).

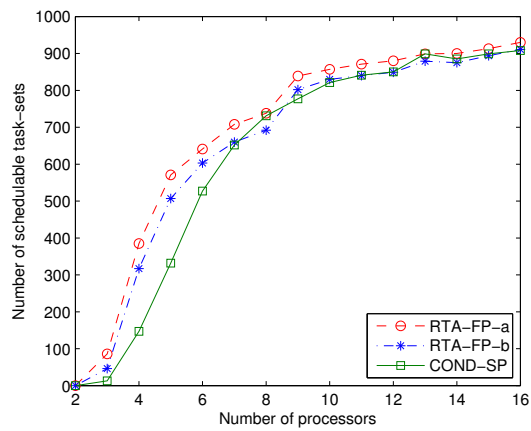

Figure 11: RTA-FP as a function of $m$ $\left(U_{\mathcal{T}}=2\right.$, constrained deadlines).

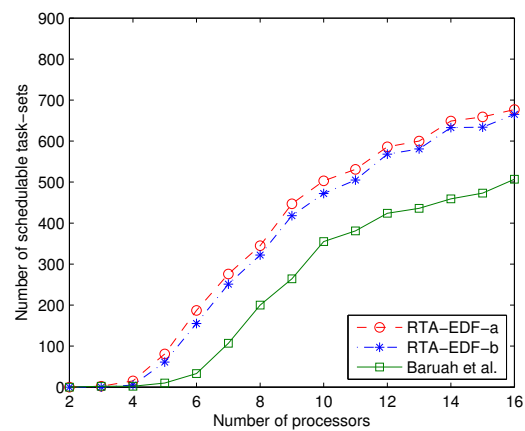

Figure 14: RTA-EDF as a function of $m$ $\left(U_{\mathcal{T}}=2\right.$, constrained deadlines).

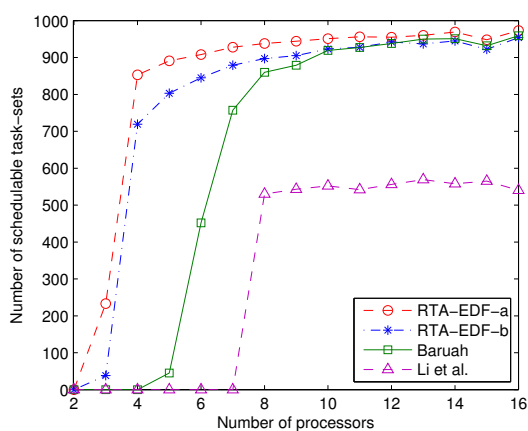

Figure 17: RTA-EDF as a function of $m$ ( $U_{\mathcal{T}}=2$, implicit deadlines).

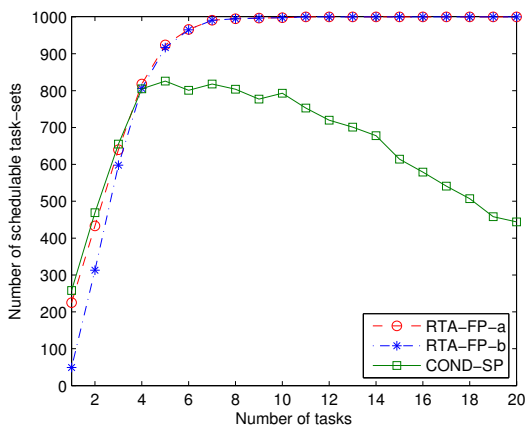

Figure 12: RTA-FP as a function of $n$ ( $m=4, U_{\mathcal{T}}=2$, constr. deadlines).

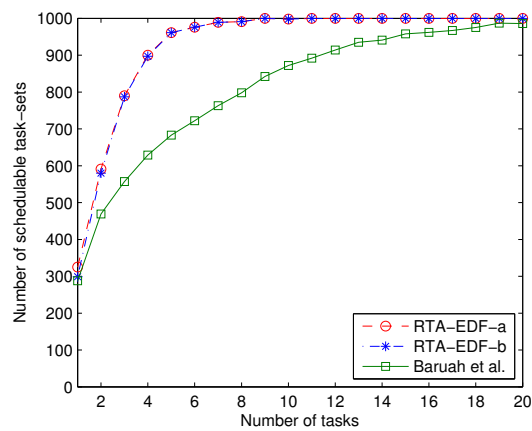

Figure 15: RTA-EDF as a function of $n$ ( $m=8, U_{\mathcal{T}}=2$, constr. deadlines).

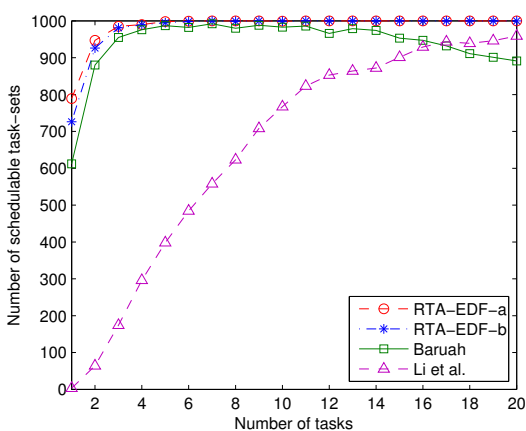

Figure 18: RTA-EDF as a function of $n$ ( $m=8, U_{\mathcal{T}}=2$, implicit deadlines). time analysis for global EDF over existing schedulability tests targeting systems of sporadic DAG tasks.

The random task generator described above can be simply adapted to generate classical DAG tasks by setting $p_{\text {cond }}=0$ and requiring that $p_{\text {term }}+p_{\text {par }}=1$. Specifically, we set: $p_{\text {par }}=0.8, p_{\text {term }}=0.2, p_{\text {add }}=0.1, n_{\text {par }}=6$, $\beta=0.1$.

We compared our RTA-EDF test against three schedulability tests for systems of sporadic DAG tasks scheduled according to global EDF:

- the test by Baruah [9], which analytically dominates the one in [15];

- the test by Li et al. [23], based on capacity augmentation bound;
- the test by Qamhieh et al. [29] that takes into account the internal structure of the DAG.

Since the test in [23] assumes implicit deadlines, we report all the results under that setting for consistency, although similar results have been obtained also in the general case of constrained deadlines. We do not plot the results of the test in [29], because its performance was very poor in all observed configurations. This stems from the fact that the analysis in [29] is mainly focused with improving the minimum processor speed that guarantees schedulability under global EDF, rather than ensuring a good schedulability performance.

Figure 16 illustrates the number of schedulable task-sets in the case of $m=8$ and varying utilization $U_{\mathcal{T}} \in[0,8]$. 
While the performance of RTA-EDF drops around $U_{\mathcal{T}}=5$, the breakdown utilization of the other approaches is significantly lower.

Figure 17 illustrates the performance of RTA-EDF when $U_{\mathcal{T}}=2$ and $m$ is varied in $[2,30]$. As evident from the figure, RTA-EDF substantially outperforms the other tests, as it requires a significantly lower number of cores (around 5) to schedule most task-sets, while the test in [9] typically requires twice that number, and it cannot admit any task-set when $m<5$. The behavior of the test in [23] is even worse, since a large share of the generated task-sets are not deemed schedulable even if a very large number of cores is available. This result indeed reflects the analytical formulation of the test given in [23].

Figure 18 reports the results obtained when varying the number of tasks $(n \in[1,20])$, with $m=8$ and $U_{\mathcal{T}}=2$. As before, our approach substantially outperforms the others for any value of $n$. The test in [9] shows a slowly degrading trend for high values of $n$. Instead, the one in [23] is favorably impacted by increasing $n$, since by keeping the total utilization constant the individual critical path lengths are reduced in average, which is beneficial for the outcome of the test.

This class of experiments clearly shows that the effectiveness of our schedulability analysis goes beyond conditional task structures, as it is able to significantly tighten the schedulability of non-conditional DAG task systems as well.

\subsection{Evaluation of RTA-FP vs. RTA-EDF}
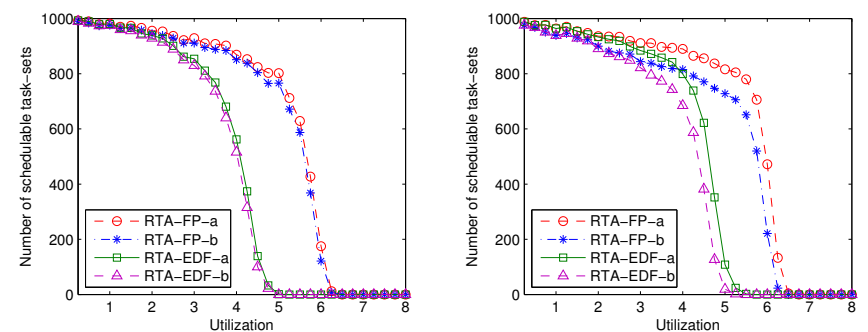

Figure 19: RTA-FP/EDF as Figure 20: RTA-FP/EDF as a a function of $U_{\mathcal{T}}$ (cp-tasks, function of $U_{\mathcal{T}}$ (DAGs, $m=$ $m=8$, implicit deadlines). $\quad 8$, implicit deadlines).

We now compare the performance of our response-time analysis for global FP (with priorities assigned by DM) and global EDF as a function of the system utilization $U_{\mathcal{T}}$. In the following experiments, we consider $m=8$ and restrict to the case of implicit deadlines. Figure 19 and 20 report the results for the case of cp-tasks and classic DAG tasks, where the parameters for the task generation have been set as in Section 6.1 and 6.2, respectively. In both cases, RTA-FP is able to guarantee $\sim 75 \%$ of the total system utilization, being able to detect a significant amount of schedulable task-sets until $U_{\mathcal{T}}=6$, while the performance of RTA-EDF drops at a smaller utilization level (around $U_{\mathcal{T}}=5$ ), which corresponds to $\sim 63 \%$ of the total system utilization.

As a salient trait, in both cases the performance of the schedulability test for global FP is significantly superior to the corresponding test for EDF, resembling the behavior observed in the case of multiprocessor response-time analysis for sequential task systems [12]. This is mainly due to the fact that in the case of FP the interference from lower-priority tasks can be neglected, which does not hold when EDF is used. Therefore, the schedulability performance achieved by our RTA-FP test is able to largely compensate the disadvantage of FP vs. EDF in terms of absolute scheduling performance.

As a final remark, we observe that the performance of our response-time analysis in the conditional case (Figure 19 ) is very similar to the case when no conditional vertices are assumed (Figure 20). This fact leads to the conclusion that the concept of worst-case workload is a meaningful characterization of conditional-parallel real-time workload, and, more in general, that the presented analysis is able to effectively integrate conditional statements with parallel execution flows.

\section{Conclusions}

This paper considered a new task model, the cp-task model, that generalizes the classic sporadic DAG task model by integrating conditional branches. Such an additional information is exploited by the schedulability analysis to derive a tighter estimation of the interfering contributions, by discriminating their level of parallelism depending on the conditional path undertaken. The topological structure of a cp-task graph has been characterized in terms of two recursive composition rules. Then, a schedulability analysis has been derived to compute a safe upper-bound on the response-time of each task in pseudo-polynomial time. Besides its reduced complexity, the proposed analysis has the advantage of requiring only two parameters to characterize the complex structure of the conditional graph of each task: the worst-case workload and the length of the longest path. Algorithms have also been proposed to derive these parameters from the DAG structure in polynomial time.

Schedulability experiments carried out with randomly generated cp-task workloads clearly show that the proposed approach does not only improve over previously proposed solutions for conditional DAG tasks, but can also be used to significantly tighten the schedulability analysis of classic (non-conditional) sporadic DAG task systems.

\section{ACKNOWLEDGMENTS}

This work has been supported in part by the European Community under the JUNIPER project (FP7-ICT-2011.4.4), grant agreement n. 318763, and the P-SOCRATES project (FP7/2007-2013), grant agreement n. 611016. The authors also like to acknowledge Risat M. Pathan for detecting a (minor) rounding error in an earlier version of Theorem 5.1, as well as the anonymous reviewers for their suggestions.

\section{RefERENCES}

[1] Kalray. http://www.kalrayinc.com/.

[2] Parallela. http://www.parallela.org/.

[3] Texas Instruments. The 66AK2H12 Keystone II Processor. http://www.ti.com/product/66AK2H12.

[4] A MATLAB@ implementation of schedulability http:/ / retis.sssup.it/ al.melani/downloads/cptasks.zip, 2015. 
[5] M. Anand, A. Easwaran, S. Fischmeister, and I. Lee. Compositional feasibility analysis of conditional real-time task models. In ISORC, 2008.

[6] B. Andersson and D. de Niz. Analyzing global-EDF for multiprocessor scheduling of parallel tasks. In OPODIS, 2012.

[7] P. Axer, S. Quinton, M. Neukirchner, R. Ernst, B. Döbel, and H. Härtig. Response-time analysis of parallel fork-join workloads with real-time constraints. In ECRTS, 2013.

[8] S. Baruah. Feasibility analysis of recurring branching tasks. In EMWRTS, 1998.

[9] S. Baruah. Improved multiprocessor global schedulability analysis of sporadic DAG task systems. In ECRTS, 2014.

[10] S. Baruah. The federated scheduling of systems of conditional sporadic DAG tasks. In EMSOFT, 2015.

[11] S. Baruah, V. Bonifaci, A. Marchetti-Spaccamela, L. Stougie, and A. Wiese. A generalized parallel task model for recurrent realtime processes. In RTSS, 2012.

[12] M. Bertogna and M. Cirinei. Response-time analysis for globally scheduled symmetric multiprocessor platforms. In RTSS, 2007.

[13] E. Bini and G. C. Buttazzo. Measuring the performance of schedulability tests. Real-Time Systems, 30(1-2):129-154, 2005.

[14] B. Blackham, M. H. Liffiton, and G. Heiser. Trickle: Automated infeasible path detection using all minimal unsatisfiable subsets. In RTAS, 2014.

[15] V. Bonifaci, A. Marchetti-Spaccamela, S. Stiller, and A. Wiese. Feasibility analysis in the sporadic DAG model. In ECRTS, 2013.

[16] A. Burns and S. K. Baruah. Sustainability in real-time scheduling. Journal of Computing Science and Engineering, 2(1):74-97, 2008.

[17] S. Chakraborty, T. Erlebach, and L. Thiele. On the complexity of scheduling conditional real-time code. In WADS, 2001.

[18] H. S. Chwa, J. Lee, K.-M. Phan, A. Easwaran, and I. Shin. Global EDF schedulability analysis for synchronous parallel tasks on multicore platforms. In ECRTS, 2013.

[19] S. Dasgupta, C. H. Papadimitriou, and U. V. Vazirani. Algorithms. McGraw-Hill, 2006.

[20] J. C. Fonseca, V. Nélis, G. Raravi, and L. M. Pinho. A multiDAG model for real-time parallel applications with conditional execution. In SAC, 2015.

[21] K. Lakshmanan, S. Kato, and R. Rajkumar. Scheduling parallel real-time tasks on multi-core processors. In RTSS, 2010.

[22] S. Lee and M. M. Crawford. Unsupervised multistage image classification using hierarchical clustering with a bayesian similarity measure. IEEE Transactions on Image Processing, 14(3):312-320, March 2005.

[23] J. Li, K. Agrawal, C. Lu, and C. Gill. Analysis of global EDF for parallel real-time tasks. In ECRTS, 2013.

[24] J. Li, J. Chen, K. Agrawal, C. Lu, C. D. Gill, and A. Saifullah. Analysis of federated and global scheduling for parallel real-time tasks. In ECRTS, 2014.

[25] J. Li, Z. Luo, D. Ferry, K. Agrawal, C. Gill, and C. Lu. Global EDF scheduling for parallel real-time tasks. Real-Time Systems, 51(4):395-439, 2015.

[26] C. Maia, M. Bertogna, L. Nogueira, and L. M. Pinho. Responsetime analysis of synchronous parallel tasks in multiprocessor systems. In RTNS, 2014.

[27] G. Nelissen, V. Berten, J. Goossens, and D. Milojevic. Techniques optimizing the number of processors to schedule multi-threaded tasks. In ECRTS, 2012.

[28] B. Peng, N. Fisher, and M. Bertogna. Explicit preemption placement for real-time conditional code. In ECRTS, 2014.

[29] M. Qamhieh, F. Fauberteau, L. George, and S. Midonnet. Global EDF scheduling of directed acyclic graphs on multiprocessor systems. In RTNS, 2013.

[30] A. Saifullah, K. Agrawal, C. Lu, and C. D. Gill. Multi-core realtime scheduling for generalized parallel task models. In RTSS, 2011.

[31] A. Saifullah, D. Ferry, J. Li, K. Agrawal, C. Lu, and C. D. Gill. Parallel real-time scheduling of DAGs. IEEE Trans. Parallel Distrib. Syst., 25(12):3242-3252, 2014.

[32] J. Valdes, R. E. Tarjan, and E. L. Lawler. The recognition of series parallel digraphs. SIAM Journal on Computing, 11(2):298-313, 1982.

[33] R. Vargas, S. Royuela, M. A. Serrano, X. Martorell, and E. Quiñones. A lightweight OpenMP4 run-time for embedded systems. In ASP-DAC, 2016.

[34] P. Viola and M. Jones. Rapid object detection using a boosted cascade of simple features. In CVPR, 2001. 